\title{
Creating cyberspace in the Solar System: Future Human Image
}

\author{
Sergey Sukhonos \\ Candidate of Technical Sciences, Russian Philosophical Society \\ (Moscow, Russia) \\ E-mail: ssuhonos@mail.ru \\ https://orcid.org/0000-0001-8710-2111
}

\begin{abstract}
In the article, the author considers the possibility of creating cyberspace in the Solar System. A new concept for the development of the Solar System using cybernetic systems and robots, which will be controlled by the new formation from Earth and partly with the help of inspection flights and commissioning, is proposed. This is a "cosmic civilization" considering the near space as a sphere of everyday self-realization. The evolutionary, social and technological aspects of the emergence and development of cyberspace are outlined. The evolutionary futility of space exploration using biological organisms is shown.
\end{abstract}

Keywords: cybernetic organism, cyberspace, evolutionary leap, cosmic civilization, space exploration

Received: September 11, 2019; accepted: September 30, 2019

Future Human Image, Volume 12, 2019: 85-93.

https://doi.org/10.29202/fhi/12/7

\section{Introduction}

In the twentieth century, humankind dreamed of space and in the second half of that century, the most significant steps in its study and development were taken. However, by the end of the twentieth century, the pace of space exploration fell sharply [Krichevsky, 2013; 2017]. Astronauts do not fly further than the International Space Station. A lot has been written about the Moon and Mars in the media, but there are no more real projects for their development at present.

The main reason for the decline in interest in space exploration is the difficulty of human staying in the outer space. These difficulties can be reduced to four obvious factors: radiation, weightlessness, the environment of outer space that is unsuitable for living (low temperature, vacuum, etc.), and the inability to grow high-grade products in small ecosystems. The latter was shown by the Biosphere- 2 experiment. That is why many astronauts, who spent long time in orbit, claim that man has no place in space.

It is beyond argument that all these problems can be solved in course of time. For example, by creating genetically modified organisms for which radiation will not be scary. Oleg Konon-

(C) Sukhonos, Sergey, 2019 
enko, the commander of the Russian astronaut team, believes that genetically modified people will explore deep space. He said this at a "round table" at Bauman Moscow State Technical University [Russian cosmonaut, 2019]. It is possible that with the large sizes of space stations and due to their rotation, it will be possible to create artificial gravity. It is likely that biotechnology in the future will allow the cultivation of meat and bread with the help of bacteria, and the bacteria will be fed by radiation and other types of emissions. But in any case, the main problem remains - this is the hostile environment of the cold cosmic vacuum. Therefore, astronauts will still have to live inside sealed capsules of different sizes.

With respect to these problems, the question arises "Why does man need space?" To colonize planets in order to save the Earth from overpopulation? But the exploration of the Moon, and especially Mars, for this purpose looks like utopia. Moreover, thousands of people, but not millions, will be able to live there at a high cost. This does not solve the problem of overpopulation of Earth with billions of people. Everything is clear with the near-Earth space Earth observation, communications, etc., almost everything in this domain can be successfully solved by means of automated equipment.

The only pragmatic task that justifies manned flights, and which is beyond doubt, is the creation of space energy to solve environmental and energy problems that have been simmering on the planet. First of all, the question is about the collection of solar energy and its transfer to Earth (Fig. 1).



Fig. 1. The scheme of collection and processing of solar radiation

In the twentieth century, such stations were planned to be created with the help of people. However, in the twenty first century, thanks to the information-cybernetic revolution, the possibility of using robots and cybernetic organisms for this purpose is quite visible. We are faced with a global contradiction in the ideology of space exploration. The twentieth-century romance by inertia draws us to manned flights, and the twenty-first century rationalism leads to the transfer of this mission to robots and cybernetic organisms. 
To gain insight into this issue, it is necessary to look at the development of life from the highest heights of its evolutionary path [Sukhonos, 2007].

\section{Global stages of the evolution of the biosphere and humanity in the phase spaces of the planet and outer space}

To begin with, life on Earth originated in the ocean in the form of unicellular organisms. Two billion years later, it spread on land. Hundreds of millions of years passed, and it flew up into the sky — birds appeared. In this way, within three billion years, life has gradually mastered the three phase states of matter - liquid, solid and gaseous.

Physics knows another phase state - plasma. This state of matter is rarely found on Earth. However, in outer space, more than $90 \%$ of the substance is in the plasma state. The stars are predominantly spheres of plasma. Therefore, the next step in life evolution is obvious. This is the exploration of plasma and the outer space.

Is it a coincidence that several million years ago man survived in the wild, primarily because they first mastered the fire, and then began to use fire in everyday activities? Physics call fire as a degenerate state of plasma. The global goal of humankind in the field of new energy is the creation of a thermonuclear plasma controlled reactor (experiments have been going on for about 70 years).

Man is the only mammal on the planet that is not only not afraid of fire, but uses it in its activities, gradually increasing its power. The legend of Prometheus in the ancient Greek interpretation indicates that it was fire, which Prometheus purposefully presented to people so that they could protect themselves from the wild animals and cold. The name of the best Soviet film about space exploration "Taming the Fire" can be considered symbolic.

Using the extrapolation method, it can be assumed that life as a planetary phenomenon, which, according to the thesis of Vladimir Vernadsky, seeks to fill with itself all the spaces and media accessible to it, having mastered the three phase states of matter, took a new evolutionary step. It created man by arming them with fire and external energy. Life created man with the goal of walking into outer space.

In the middle of the twentieth century, man walked in space, having previously mastered the land, oceans, and only a little more than a hundred years ago, they got off the ground into the air (Fig.2).

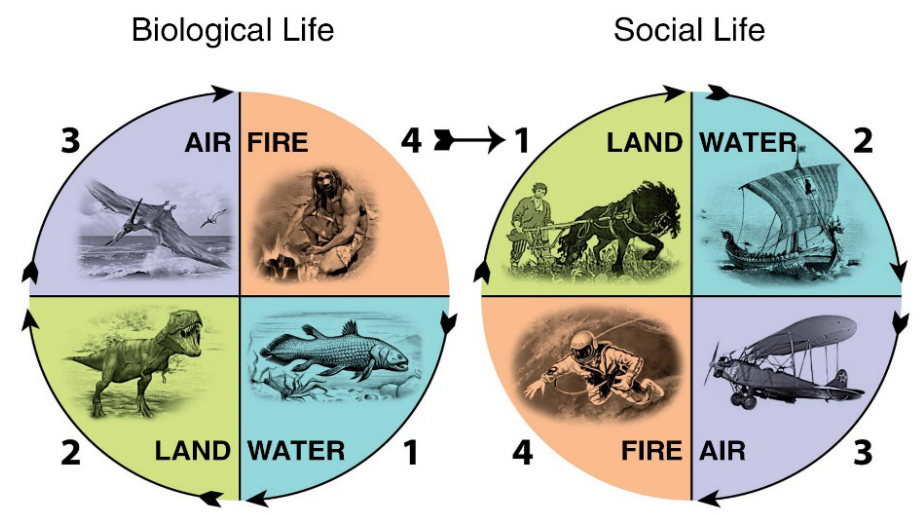

Fig. 2. The exploration of the four phase spaces by the biosphere and man 
If you rely on the global trend of the life development on Earth, it is necessary to recognize that humanity did not appear on Earth in order to create a paradise for itself on the planet. Man appeared so that the evolution of life could step into outer space. This is the mission and purpose of humankind!

Consider this issue in more detail.

The fact is that the exit of man into space in a biological body is problematic. The main doubt is whether people will be able to carry out space exploration "inside" their biological organisms, which evolutionarily arose for life on land and are completely unsuitable for life in outer space.

\section{The inevitability of the evolutionary transformation of man in the process of space exploration}

Briefly consider the evolution of life on the planet. When the development of life became limited in the oceans and seas, it came onto land not in the form of fish, but through gigantic evolutionary work. It created a number of land animal species, most of which are not adapted to life in the ocean. Evolution did a similar transformation when life entered the airspace.

Therefore, to explore the new environment, evolution must create a fundamentally new body. The best option seems to be a cybernetic organism. Highly developed cybernetic organisms are not afraid of radiation and cosmic cold. Perhaps they will be able to be "fed" by solar radiation, or due to the energy of small nuclear reactors. In addition, weightlessness will even facilitate their construction.

In 2019, Russia launched the Fedor robot into space. Of course, modern robots are still far from the state, in which they can be entrusted with self-dependent work in space, and even more, be considered as highly developed cybernetic organisms. However, the world of artificial intelligence and the functionality of robots is rapidly evolving. It can be assumed that over time, outer space, at least within the asteroid belt, will gradually become "populated" with more advanced robots and cybernetic organisms.

In this case, the question arises "What will happen to humanity?"

Assume the following future development scenarios:

1. The pessimistic scenario. Man becomes an intermediate stage in the development of life between Earth and space. Its mission is limited to creating cyberspace and artificial intelligence in outer space. A man must give cyberspace a primary impetus.

2. The optimistic fantastic scenario. Man will temporarily engage in the development of the Universe in a biological body. After a certain period, there will be a global transformation of their state into a new form, e.g. polar or radiant (according to Konstantin Tsiolkovsky). In this form, the existence of man will no longer depend on technical support. In this case, robots and cybernetic organisms will remain as a side branch of evolution within the Solar System.

3. The evolutionary scenario. In the history of culture, the development of technical means and travelling speed occurred irregularly. Each leap led to a substantial increase in the travelling speed. From a horse to a rocket, several leaps were made. It is logical to assume that the next giant leap for humankind awaits. For example, the discovery of a method of almost instantaneous transfer to anywhere in the Universe. In this case, man will be able to settle on other planets that have a suitable environment for life without difficult and lengthy flights through outer space. The 
need for the body transfiguration and its transformation into a cybernetic organism will disappear. Robots will be mere assistants in such a resettlement.

\section{Space robotization as a way to solve the urgent needs of humankind}

Nowadays, the creation of cyberspace in the Solar System is a reality. Modern technology leads humanity along the path of merging the capabilities of high technology and neuroscience. In creating cyberspace, at least four stages of development can be distinguished.

1. Creation of a class of robots capable of independent access to space and independent work in it. This phase will complete with the creation of a class of robots capable of staying in outer space at an ISS-type station. They will carry out external repair works of the station, as well as installation and maintenance operations.

2. Creation with the help of robots of power stations based on the collection of solar radiation with subsequent transfer of energy to Earth. Robots will also specialize in the station maintenance and expansion.

3. Creation of a "settlement" of robots on the Moon, as well as factories for the extraction and processing of useful resources from its surface. The self-reproduction of robots and the production of separate parts by robots for their own configuration are supposed. Such a settlement will partly be capable of independent "breeding" of robots or reproduction of analogues for further exploitation on the Moon or during the development of other planets of the asteroid belt.

4. Complete technical exploration of the Solar System within the asteroid belt by robots.

It is obvious that the more diverse in quality and quantity is the classification of robots, the more people will be involved in working with them. In addition, here we can distinguish several areas of cyberspace development.

1. Scientific and technical work, which provides for the development of systems of robots and their control. Scientific and technical personnel will be needed.

2. Pilot production of the first robots. Highly qualified engineers, workers and installers will be in demand.

3. Training and maintenance of robots in outer space, control over their work. Astronaut engineers in orbit, as well as cybernetic organisms, will be required.

4. Remote tracking of robots in their activities from the ground. The most capable remote control operators will be needed.

All these activities on Earth and in the near space will involve an increasing number of people. This is the way, according to Sergey Krichevsky, to the creation of a space state [Krichevsky \& Udartsev, 2019].

At the first stages, robots in the created cyberspace will have to be controlled from Earth or from orbit. Consequently, man on Earth will increasingly develop the functions of an avatar of cyber systems in space. The more the robots will free people from working on Earth, the more people will be able to realize themselves in the cyberspace created in outer space. 


\section{Why does humanity need cyberspace in outer space?}

The question of the practical feasibility of creating cyberspace in the near space is a question of the need to consume clean energy instead of "dirty technologies" on Earth. The higher is the threat of environmental disaster on Earth, the more acute the question about creating space energy will be, and, accordingly, cyberspace in outer space.

Creating cyberspace in space will require tremendous expenses from humanity that is obvious. However, another thing is obvious. After the robots learn to reproduce themselves from space materials extracted, e.g. on the Moon or on asteroids, their world will become self-sufficient and profitable for all people. Robots will consume free solar energy and use extraterrestrial sources of raw materials. In return, they will supply humanity with pure cosmic energy, and, if necessary, with raw materials.

The creation of cyberspace in outer space is most likely to encounter inexplicable natural phenomena, the analysis of which will require the presence of man. Therefore, the most courageous people will accompany robots in space and help them create cyberspace in outer space. The most creative part of humanity will be engaged in the creation of new technologies on Earth for those working in space. A variety of activities aimed at creating cyberspace will lead to the creation of "cosmic humanity."

\section{The fourth stage in the development of earthly life - cosmic}

Consider the philosophical aspect of creating cyberspace in the Solar System.

Life, as it developed, performed grandiose transitions from one level of the hierarchy to another (Fig. 3-5). Each of its steps is a tremendous transformation not only from the point of view of a new environment, but also of new organisms. The first level was occupied by the world of unicellular organisms - from viruses to amoebas. The second level was taken by multicellular organisms from small crustaceans to whales and sequoias. It is important to note that fundamentally new biological structures — social systems - arose on the third M-level (Sukhonos, 2014).

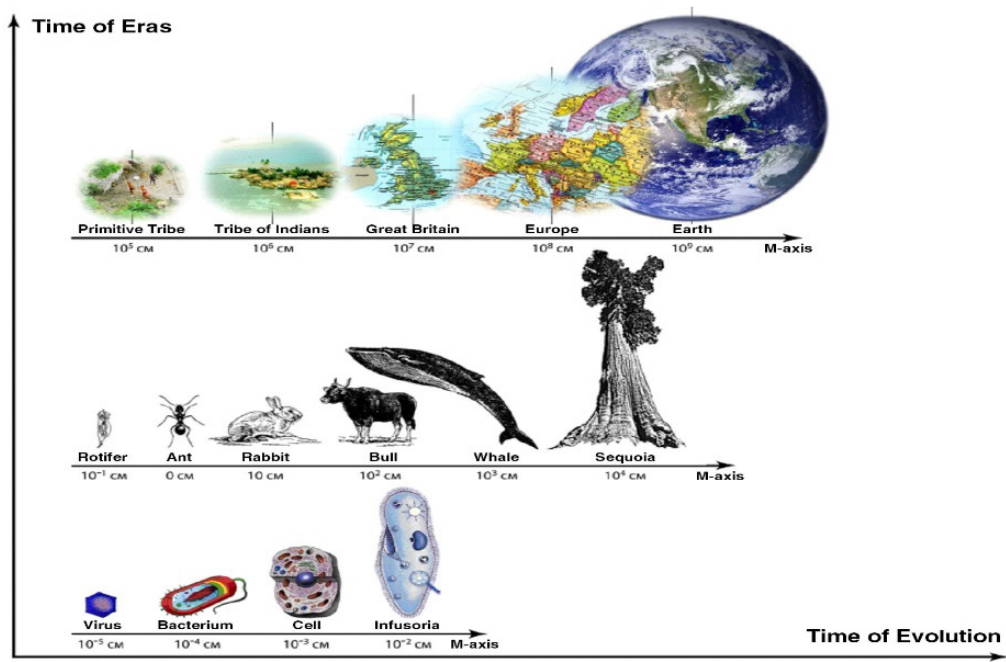

Fig. 3. Three global stages of the evolution of life on Earth 
The evolution of living organisms can be clearly divided into three large-scale stages, or into three large-scale levels: unicellular, multicellular, and social systems-organisms.

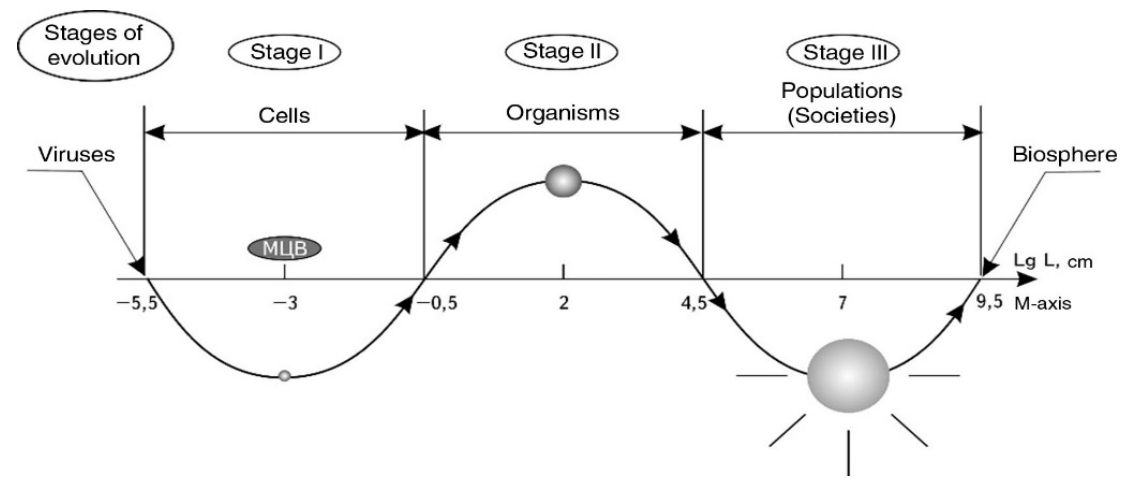

Fig. 4. Three consecutive stages of the evolution of life along the axis

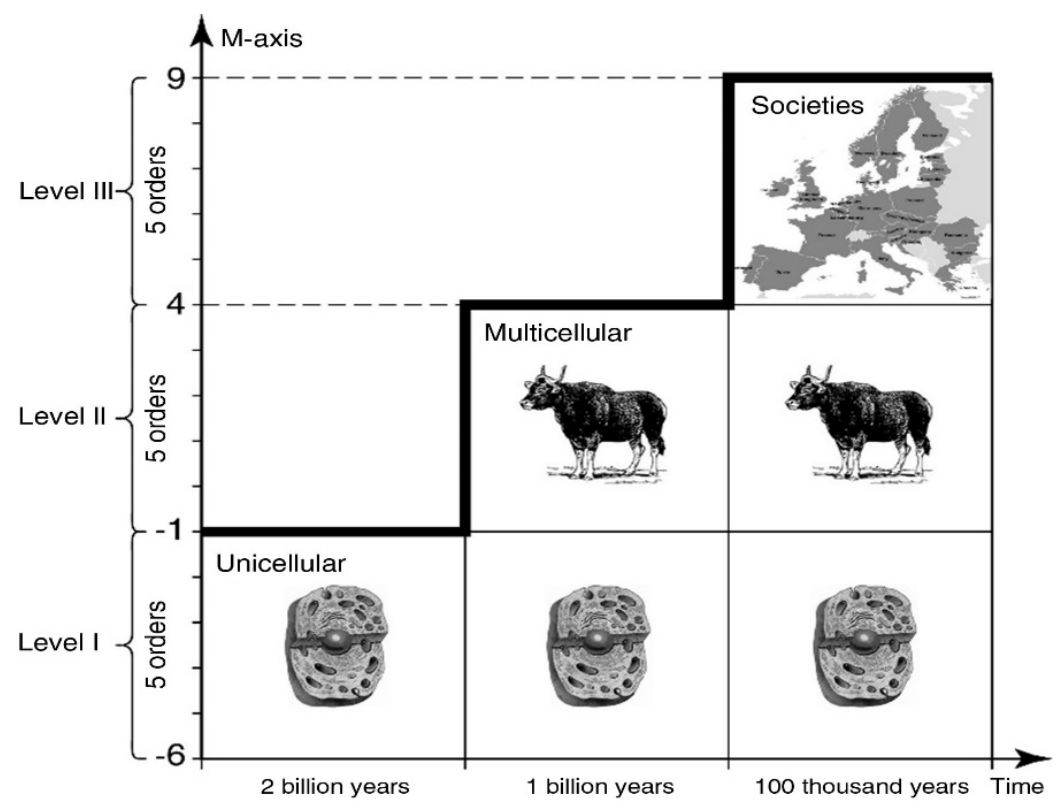

Fig. 5. Three steps of ascent of living creatures on a large-scale "ladder"

All three stages of the development of life along the axis of the development of the Universe can be represented in the form of three stages of ascent from unicellular through multicellular to social systems-organisms. Each stage can conditionally be called a large-scale level, and each stage occupied much shorter period of time in evolution.

Going beyond the borders of the planet and creating cyberspace within the asteroid belt will lead to the "settlement" of the fourth scale interval of the same length by 5 orders of mag- 
nitude (Fig. 6), as well as the previous ones. Thus, the world of unicellular organisms spawned the world of multicellular ones, the world of multicellular organisms spawned the world of societies, and the world of societies is on the verge of the era of cyberspace creation, which covers the near space.

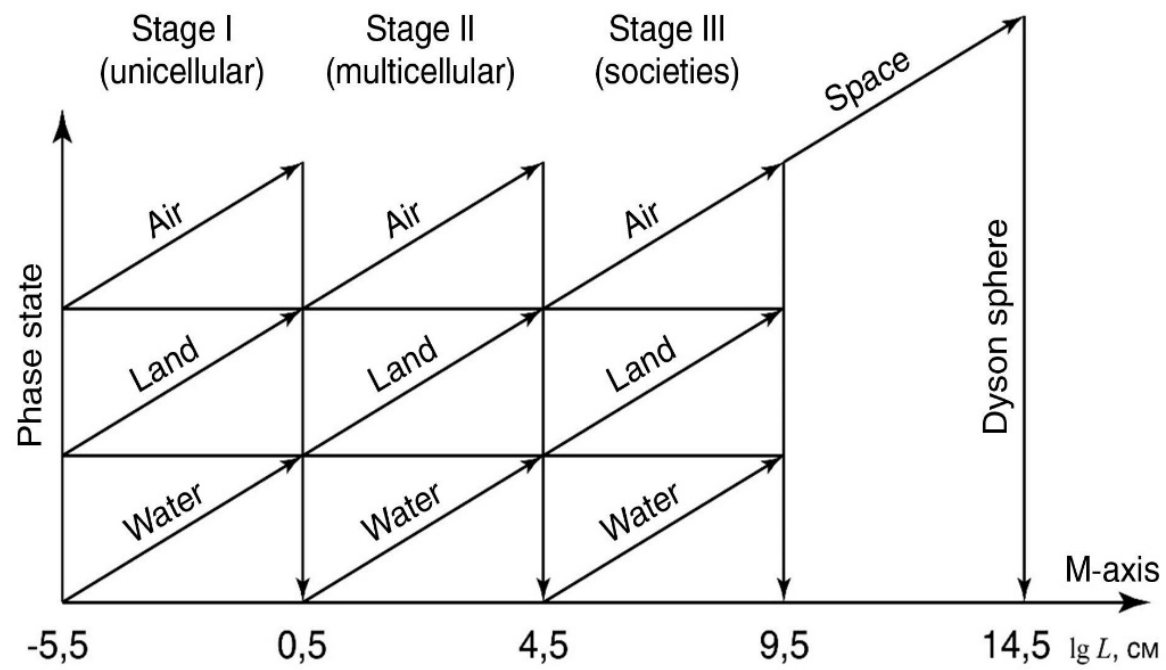

Fig. 6. The fourth cosmic stage of the large-scale evolution of life.

From the author's point of view, space exploration with its resources should lead to the formation of a special cosmic civilization on Earth that will rely on a completely different resource and technological base.

\section{Conclusions}

1. The logic of the entire evolution of life on Earth shows that humanity appeared in order to bring life into space.

2. So far, space access has become possible solely with the help of technical means and purposeful unification of large states, which represent the world of societies.

3. Societies are not just the number of people, but individual living creatures, which in their sizes occupy the third large-scale stage of life evolution, towering above the second level of multicellular ones.

4. The development of new phase media and new environments during the evolution of life on Earth has always been accompanied with a radical change in the types of organisms. For each of the three environments, evolution has created an optimal set of the sum of species that is best suited for this particular environment - water, land and air.

5. Going into outer space and mass exploration of the fourth phase medium is impossible with the help of biological organisms; therefore, the only option for "populating" outer space in the Solar System is currently evident — with the help of cybernetic organisms and robots. 
6. Cyberspace in outer space will continue the expansion of life. As a result of long-term and multi-stage development, the fourth large-scale level will appear with 5 orders of heights on the scale axis of the Universe within the asteroid belt.

7. Cyberspace will provide humanity on Earth with clean energy and the necessary raw materials.

8. The creation and management of cyberspace within the Solar System will lead to the formation of a special "space community" in which people will work for space and live in space. A cosmic civilization will be formed that will unite the conflicting parts of humanity. It will become the main source of resources and new information about the universe.

\section{References}

Krichevsky, Sergey. The Cosmic Future of Man and Mankind: Problems and Prospects. Russian Journal of Philosophical Sciences. 9, 2013: 38-43.

Krichevsky, Sergey. Cosmic Humanity: Utopia, Realities, Prospects. Future Human Image. 7 , 2017: 50-70.

Krichevsky, Sergey, and Sergey Udartsev. Space State on Earth and Beyond: Philosophy, Models, Experience and Prospects. Philosophy and Cosmology, Volume 23, 2019: 3052. https://doi.org/10.29202/phil-cosm/23/4

Russian astronaut predicted the flight of genetically modified people into space. Vzgliad. October 11, 2019. URL: https://vz.ru/news/2019/10/11/1002577.html

Sukhonos, Sergey. The logic of human evolution. Moscow: Economica, 2007.

Sukhonos, Sergey. Social Development Matrix. Moscow: Delphis, 2014.

Sukhonos, Sergey. Global prospect of world's unification. September 15, 2019. Available at: https://nexusnewsfeed.com/article/consciousness/global-prospect-of-world-s-unification 